Journal of Computer Science 5 (12): 1048-1054, 2009

ISSN 1549-3636

(C) 2009 Science Publications

\title{
An "Expert System" For Stereoscopic 3D Visualization (with out the Application of Conventional Attachments to the Eyes) of Computer Acquired and Computer Generated Stereopairs
}

\author{
${ }^{1}$ N. Sandya Rani and ${ }^{2}$ V. Vaithya Nathan \\ ${ }^{1}$ Department of MCA, Rajarajeswari Engineering College, Chennai-95, Tamil Nadu, South India \\ ${ }^{2}$ Department of Computer Science and Engineering, SASTRA University, \\ Tanjore, Tamil Nadu, South India
}

\begin{abstract}
Problem statement: Though stereoscopic 3D visualization technology has made considerable progress, it was always associated with eye attachments, like wearing color glasses, wearing Polaroid glasses, using timed shutters. Approach: This has considerably retarded the popularity of 3D visualization technology. Though there are discrete references in the literature for developing stereoscopic 3D visualization without application of glasses, these methods involve transferring the attachments to the monitor, direct viewing by straining the eyes, application of color variation. Results: They have not made considerable progress in this direction. An attempt is made in this study to use computer capabilities through generic algorithms to develop the stereoscopic 3D visualization without the application of glasses overcoming the above mentioned limitations. Conclusion/Recommendations: An attempt is made in this study to develop an "Expert System" using computer capabilities to give a satisfactory 3D/depth view of $2 \mathrm{D}$ images of $3 \mathrm{D}$ environments. Algorithms are developed to process both computer acquired and computer generated stereo pairs for $3 \mathrm{D}$ visualization.
\end{abstract}

Key words: Stereopairs, colored glasses, perspective stereopairs, computer acquired image, attachments

\section{INTRODUCTION}

Many 3D visualization techniques, such as stereoscopic visualization, cross eye visualization, parallel eye visualization, perspective 3D visualization are reported in the literature. Stereopairs can be either computer acquired (scanned) images of the photographs, or computer generated pictures using computer capabilities. Perspective projection method is used for creating computer-generated stereopairs. All above methods of 3D visualization ultimately end up with the application of attachments to the eyes. This has considerably reduced the popularity of above mentioned $3 \mathrm{D}$ visualization techniques. Though literature reports that stereoscopic 3D viewing is possible without using attachments to the eyes, the attachments are transferred to the screen, which results in restricting the position of the $3 \mathrm{D}$ viewer. A few methods are also developed using sophisticated $3 \mathrm{D}$ machines which make the $3 \mathrm{D}$ viewing not comfortable and not cost effective ${ }^{[2]}$. A few methods refer to direct viewing the stereo pair through straining eyes to have a 3D view. All above methods have not considerably created a breakthrough in 3D technology. An attempt is made in this study to develop algorithms for a direct $3 \mathrm{D}$ viewing without the application of attachments to the eyes.

In our 3 dimensional world, our two eyes give us two different images. This is because the two eyes are at two different positions in space, separated by a horizontal distance of nearly $65 \mathrm{~mm}$ (distance between the two eyes of an average human being). The images as viewed by the two eyes are captured on the curved surface of the retina of the eyes and are sent to the brain. The brain accepts the small horizontal disparity between the two image and in turn gives a single image with accurate depth perception. This ability is known as stereoscopy. Hence the stereoscopic 3D visualization technique essentially consists of, sending the left eye image to the left eye and right eye image to the right eye. That is, to sum up, the left eye should see the image corresponding to the left eye only, and the right eye should see the image corresponding to the right eye 
only. Different techniques such as, use of colored glasses (red and cyan) or use of Polaroid glasses (Horizontal and vertical polarization), or use of timed shutters are in common use to send the images to the respective eyes. The application of these attachments has considerably retarded the popularity of 3D visualization techniques.

An attempt is made in this study to use computer capabilities, through suitably developed algorithms, to give a $3 \mathrm{D} / \mathrm{depth}$ feel in the $2 \mathrm{D}$ images of environments without the application of the attachments to the eyes. While developing the algorithms, the fact that "the left eye is more sensitive to the red colors than the right eye" is made use of. Further, the same treatment as given to computer acquired images is also given to computer generated images ${ }^{[1]}$. The computer generated images are based on the principles of perspective projections making use of visual ray method, which is more flexible than other methods.

\section{MATERIALS AND METHODS}

Methodology for stereoscopic 3d visualization: The stereoscopic 3D visualization technique is divided into two parts. The first part deals with the $3 \mathrm{D}$ visualization of computer acquired stereopairs (scanned photographs of 3D environment). The second part deals with the 3D visualization of computer generated $2 \mathrm{D}$ images of $3 \mathrm{D}$ objects, assumed to be positioned in space forming a 3D environment. The images are generated through suitably developed algorithms using the method of perspective projections.

Methodology for computer acquired stereopairs: Creating a stereo image means, first creating two flat images, i.e., a stereopair, one image for the left eye and one image for the right eye. These images are scanned and sent to the computer. Normally, computer images are displaced on color screens and these screens use Red, Green, Blue (RGB) system to create the color of each pixel of the image. This means that all computer images are made of 3 bands, a red one, a green one and a blue one. Suitable algorithms are reported in the study and developed to identify selected color bands from each image. The red band is selected from the image corresponding to the left eye, the green and blue (cyan color) bands are selected from the image corresponding to the right eye and are shown separately. These two images are glued together to give a single image. This image is called as red-cyan image.

The conventional method of 3D visualization consists of wearing a pair of glasses, with red glass for the left eye and cyan (green and blue) glass for the right eye and viewing the above glued red-cyan image. It is reported in literature that left eye is more sensitive to red color than right eye. Further many complex processes take place in the eye retina-human brain system. All this finally results in the left eye viewing the left image alone and the right eye viewing the right image alone, resulting in a 3D feel or depth feel in the $2 \mathrm{D}$ images of the $3 \mathrm{D}$ environment ${ }^{[4]}$.

The proposed method as envisaged in this study deviates from the conventional method, after obtaining the glued red-cyan image. Computer capabilities are used to eliminate the use of attachments to the eyes and still view the 3D/depth through suitably developed algorithms as described elsewhere in this study.

In the glued image (also called as red-cyan image) there are a few red and cyan patches. In fact these patches of colors distinctly corresponds to, the depth in the $3 \mathrm{D}$ environment. It is logical to conclude, that the red patches correspond to the 3D space which the right eye could not see (if the right eye had seen, it will not appear as red but would appear as cyan). Similarly the cyan patches correspond to the 3D space, which the left eye could not see. All the space having neither red nor cyan corresponds to the 3D space, which both the left eye and right eye have seen together ${ }^{[4]}$.

At this stage, let us get back to our conventional method and use left red and right cyan glasses and analyze how the red and cyan colors are changing. A color matching is done and the values of RGB of the matched red and cyan patch pixels are recorded in the computer. A suitable algorithm is developed to assign the changed values of RGB in the patches of the glued image. Upon processing the red cyan glued image as per the developed algorithms, a fairly satisfactory $3 \mathrm{D} /$ depth feel of the 3D environment is visualized ${ }^{[3]}$.

The stereopairs used for above studies are drawn from the literature available through web under stereopairs. The reliability of the end result is tested by considering two more stereo pairs and processed as above, and reported through Fig. 1-18.

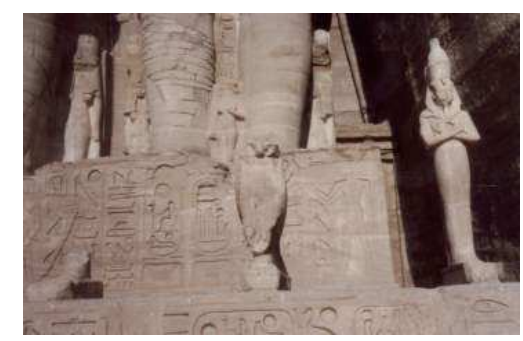

Fig. 1: Left eye image 


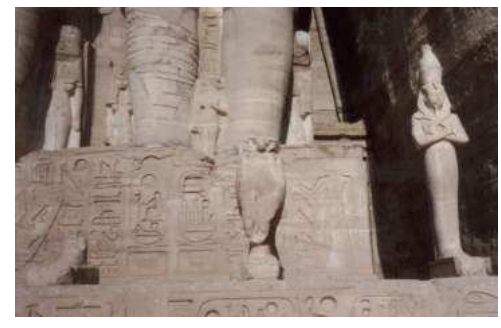

Fig. 2: Right eye image

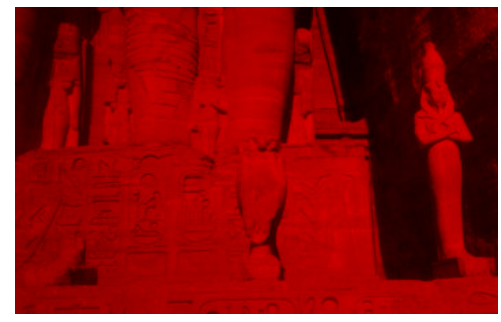

Fig. 3: Left red band image

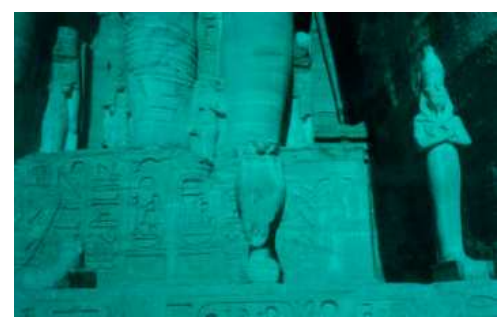

Fig 4: Right cyan band image

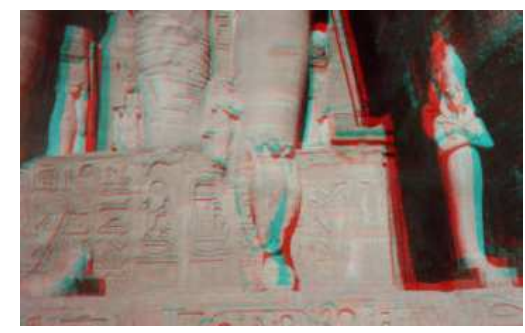

Fig. 5: Glued image

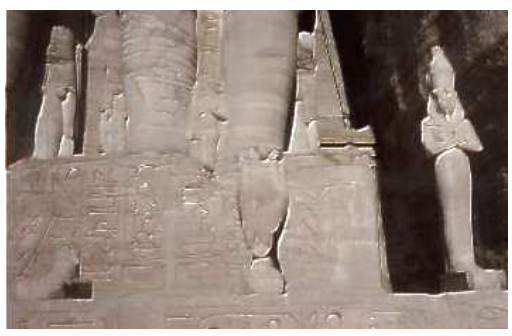

Fig. 6: Processed 3D image

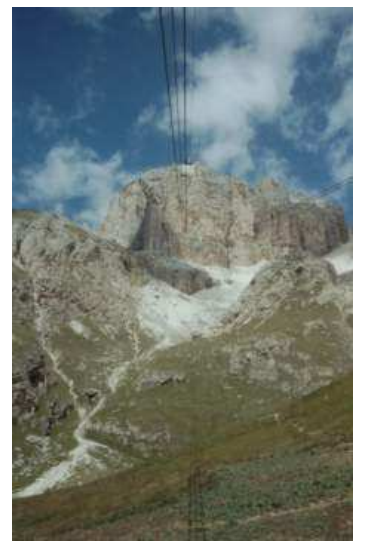

Fig. 7: Left eye image

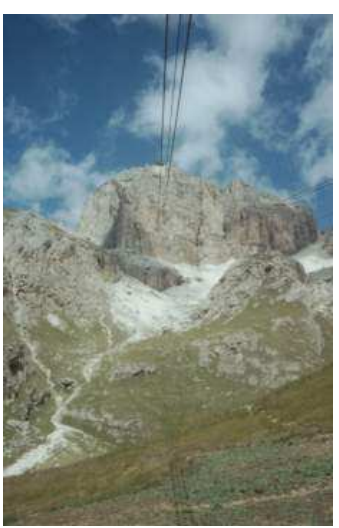

Fig. 8: Right eye image

Methodology for computer generated stereo pairs: To start with, the perspective view of a given 3D environment is obtained by choosing an optimum viewing point for a perspective view. Suitable algorithms are developed to select the optimum viewing point. Assuming that this generated image corresponds to the left eye image, the station point or the viewpoint is now shifted to right by $65 \mathrm{~mm}$ (choosing a suitable scale for convention) and again the perspective view is generated analogous to the right eye. The various surfaces of the solids appearing in both the views are given color shading based on the principles of color gradation. The processing of the left and right images is done as explained under computer-acquired images for $3 \mathrm{D}$ visualization. The conventional method of perspective views are based on the view on a flat PP. But in practice the retina of human eye on which the image is viewed has a curved surface. Hence the stereo pairs for the same 3D environment are obtained for flat, cylindrical and spherical picture planes. The usual 
processing is done for $3 \mathrm{D}$ visualization. The final processed images under spherical picture plane are considered and are reported through Fig. 19-24.

\section{Sample algorithms for acquiring red band pictures and cyan band pictures:}

Private Sub Command1_Click()

Dim red As Integer, green As Integer, blue As Integer Dim iold, inew, jold, jnew

$\mathrm{X}=$ Picture1.ScaleWidth

$\mathrm{Y}=$ Picture1.ScaleHeight

$\mathrm{p}=$ Text1.Text

$\mathrm{q}=\mathrm{Text} 2$. Text

For $\mathrm{i}=1$ To $\mathrm{Y}-2$

For $\mathrm{j}=1$ To $\mathrm{X}-2$

pixel $\&=$ Form22.Picture1.Point $(\mathrm{j}, \mathrm{i})$

red $1=$ pixel $\&$ Mod 256

green $1=(($ pixel $\&$ And $\&$ HFF00FF00 $) / 256 \&)$

blue $1=($ pixel $\&$ And \&HFF0000 $) / 65536$

ImagePixels $1(0, \mathrm{i}, \mathrm{j})=\operatorname{red} 1$

$\operatorname{ImagePixels} 1(1, \mathrm{i}, \mathrm{j})=$ green 1

ImagePixels1 $(2, \mathrm{i}, \mathrm{j})=$ blue 1

pixel $\&=$ Form22.Picture2.Point $(\mathrm{j}, \mathrm{i})$

red2 $=$ pixel $\&$ Mod 256

green $2=(($ pixel \& And \&HFF00FF00 $) / 256 \&)$

blue $2=($ pixel\& And \&HFF0000) $/ 65536$

ImagePixels $2(0, i, j)=\operatorname{red} 2$

ImagePixels $2(1, i, j)=$ green 2

ImagePixels $2(2, \mathrm{i}, \mathrm{j})=$ blue 2

Picture3.PSet (j, i), RGB(red1, green2, blue2)

Next

Next

End Sub

Sample algorithms for processing the red cyan glued picture:

Private Sub Command2_Click()

$\mathrm{X}=$ Picture1.ScaleWidth

$\mathrm{Y}=$ Picture1.ScaleHeight

For $\mathrm{i}=1$ To $\mathrm{Y}-2$

For $\mathrm{j}=1$ To $\mathrm{X}-2$

red $2=$ ImagePixels $2(0, \mathrm{i}, \mathrm{j})$

green $2=$ ImagePixels $2(1, \mathrm{i}, \mathrm{j})$

blue2 $=$ ImagePixels2 $(2, \mathrm{i}, \mathrm{j})$

If red $2>128$ And green $2>128$ And blue $2>128$ Then

Picture3.PSet (j, i), RGB(red2, green2, blue2)

End If

Next

Next

End Sub

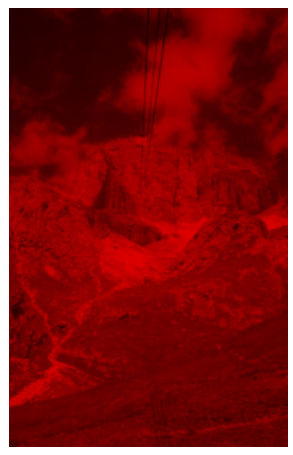

Fig. 9: Left red band image

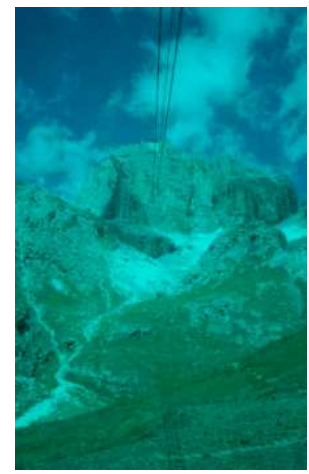

Fig 10: Right cyan band image

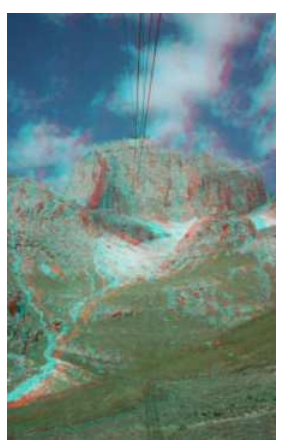

Fig. 11: Glued image

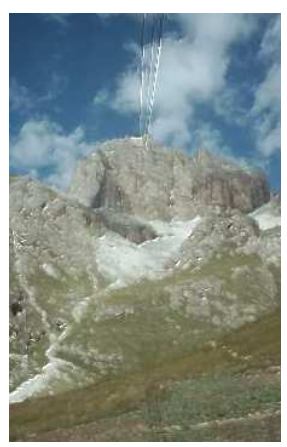

Fig. 12: Processed 3D image 


\section{Computer acquired images:}

- First set: (Fig. 1-6)

- Second set: (Fig. 7-12)

- Third set: (Fig. 13-18)

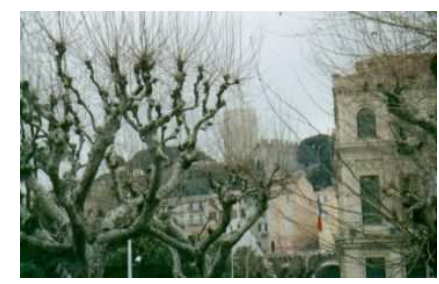

Fig. 13: Left eye image

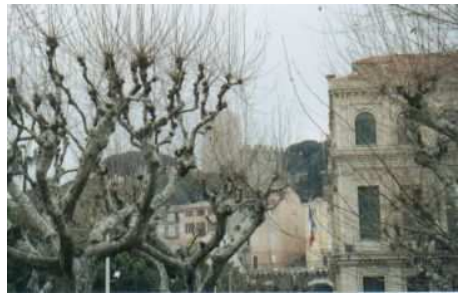

Fig. 14: Right eye image

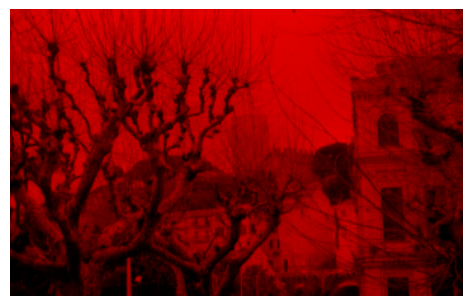

Fig. 15: Left red band image

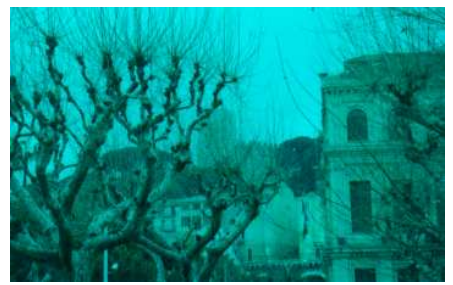

Fig 16: Right cyan band image

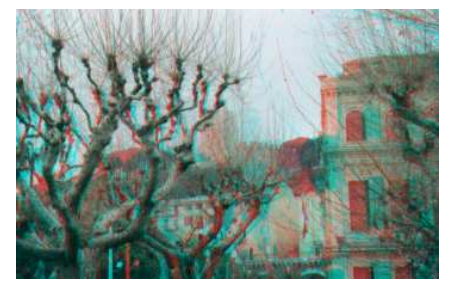

Fig. 17: Glued image

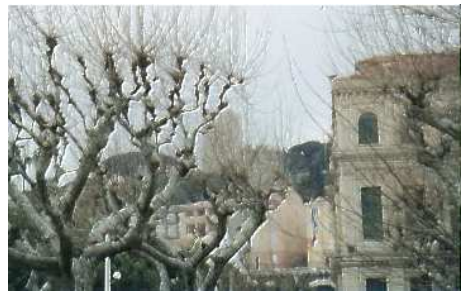

Fig.18: Processed 3D image

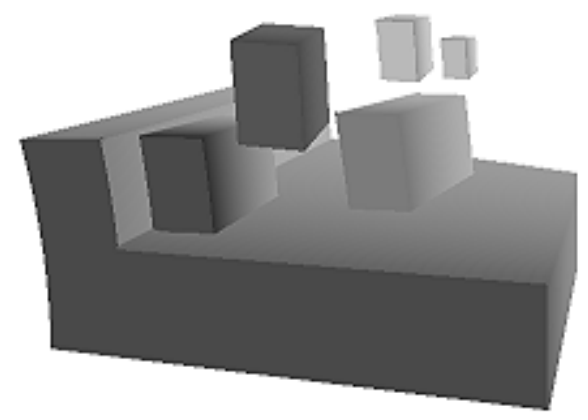

Fig. 19: Left eye image

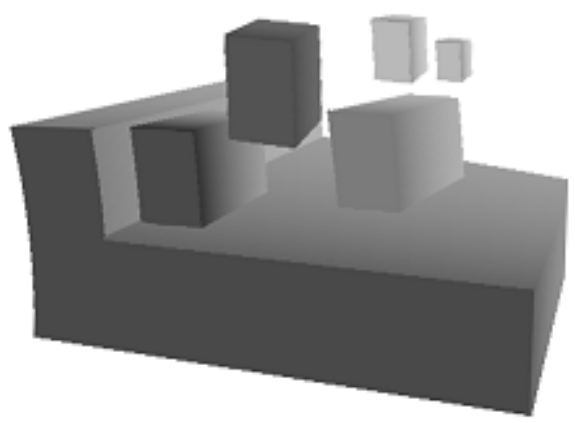

Fig. 20: Right eye image

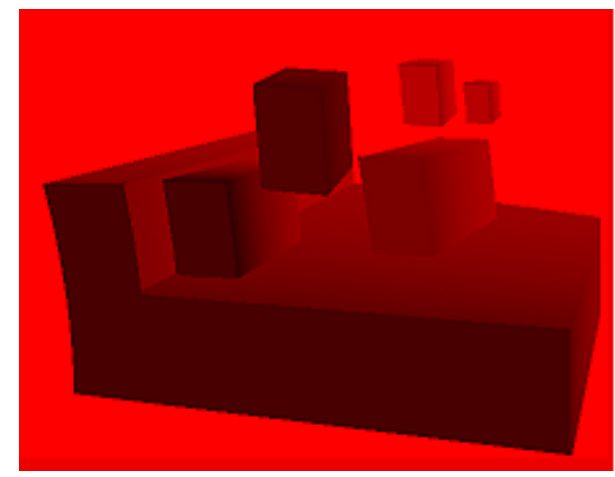

Fig. 21: Left red band image 1052 


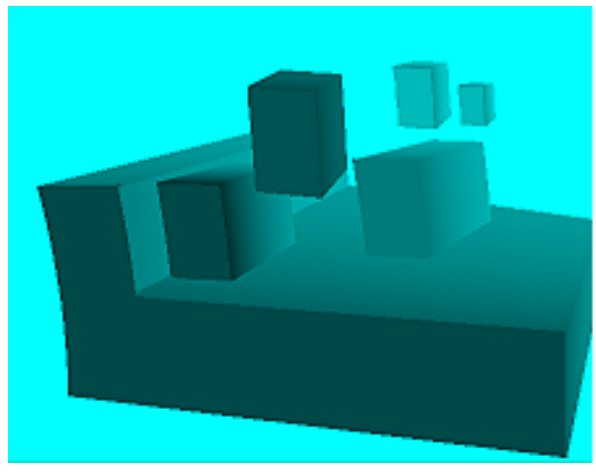

Fig. 22: Right cyan band image

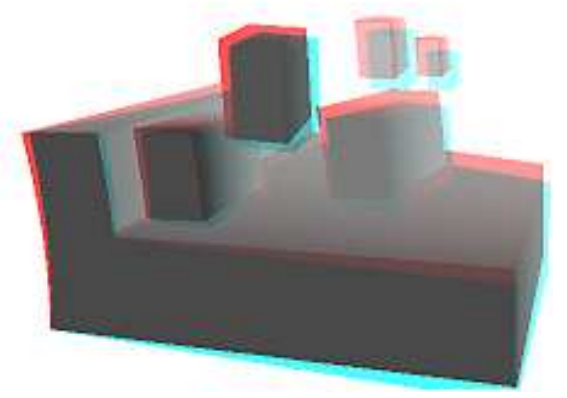

Fig. 23: Glued image

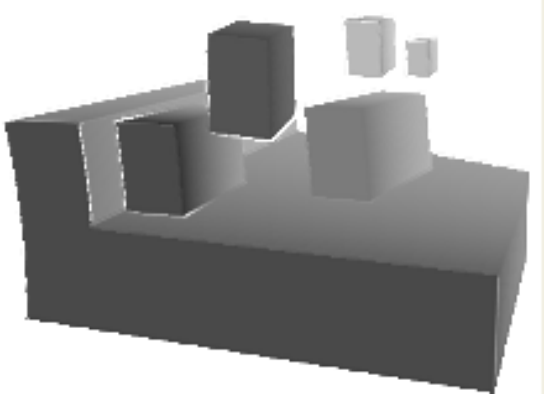

Fig. 24: Processed 3D image

Computer generated images: The generated images correspond to perspective views obtained using spherical picture plane. The method used for generating the views are based on visual ray method, using principles of perspective projections (Fig. 19-24).

\section{RESULTS}

The following results of above study are listed below:
- Both computer acquired and computer generated stereopairs are processed and they exhibit a uniform quality of $3 \mathrm{D}$ visualization

- Of the two namely acquired and generated, the generated method is more flexible. This offers an optimum position for the observer to get an ideal view of the $3 \mathrm{D}$ environment. This is possible since, different positions can be tried out using computer capabilities

- Many methods have been reported in the literature to obtain the red cyan picture. The method chosen in this study is simple because this is based on choosing the red bands and the cyan bands by developing algorithms

- The processed 3D picture when reduced in size appears to exhibit a better 3D readability

- Of the two methods available to generate perspective images namely visual ray method and vanishing point method, the visual ray method is used through out this study, since this method is more universal than the other method

- Of the three methods used for generating perspective steropairs under visual ray method, namely using flat picture plane, cylindrical picture plane and spherical picture plane, it is observed that, as we move from flat to spherical the 3D readability moves towards more realistic in nature

- The method of using spherical picture plane happens to exhibit the best 3D readability of a given $3 \mathrm{D}$ environment

\section{DISCUSSION}

- Though other methods like using Polaroid lenses for shooting the left eye and right eye images is reported in the literature, the same is not considered here due to heavy cost

- In the case of computer acquired images, the processed 3D view not only gives a good 3D readability, but also the quality and clarity of the image has considerably improved. Many details in the acquired images, which otherwise could not be seen clearly, are seen well in the 3D view

- Coming to computer generated images, which are based on principles of perspective projection, we can preposition the 3D object to give noticeable depth variation and generate the perspective stereopairs. These pairs on further processing, exhibit the required 3D depth view giving a good $3 \mathrm{D}$ view 
- Further it is observed that, (in case of computer generated stereo pairs) as the position of the observer from the picture plane increases, the 3D view moves towards more realistic in nature

\section{CONCLUSION}

From the research findings of this study the following conclusions are drawn:

- Though there are a few references in the literature regarding obtaining stereoscopic 3D views without the application of attachments to the eyes, they could not make much progress due to their inherent drawbacks as explained elsewhere in the study. The present method proposes simple generic algorithms to have a comfortable and viewer friendly 3D visualization

- The 3D views of the 2D images offer better visualization and better picture quality. The research carried out in this study concentrates on 3D visualization without the application of conventional attachments to the eyes. All said and done, ultimately we are trying to show a 3D environment on a 2D plane. Hence, a 100\% 3D effect can never be achieved. We are only trying to identify how the glued image changes to a 3D view while using attachments and simulate the same using computer capabilities and thereby eliminate the attachments

- There are no shortcuts. The left image should go the left eye and the right image should go to the right eye. Only then a $100 \%$ 3D effect can be felt. The present research concentrates on bypassing the attachments, which results 70 to $75 \%$ of 3D effect

- However, we conclude that, the day is not far off for the researchers to further develop software and hardware tools to give a 100\% 3D effect without any attachments what so ever

\section{REFERENCES}

1. Eriksson, H., 1996. Expert systems as knowledge servers. IEEE Intel. Syst., 11: 14-19. DOI: 10.1109/64.506749

2. Mizoguchi, R. and H. Motoda, 1995. Expert systems research in Japan. IEEE Intel. Syst., 10: 14-23. DOI: $10.1109 / 64.403950$

3. Sagheb-Tehrani, M., 2005. Expert systems development: Some issues of design process. ACM SIGSOFT Software Eng. Notes, 30: 1-5. http://portal.acm.org/citation.cfm?id=1050864\&dl= GUIDE $\&$ coll $=$ GUIDE $\&$ CFID $=64629342 \&$ CFTO $\mathrm{KEN}=72718524$

4. Cheung, Y., G.M. Hong and K.K. Ang, 2004. A dynamic project allocation algorithm for a distributed expert system. Expert Syst. Appli.. 26: 225-232.

http://www.pubzone.org/dblp/journals/eswa/Cheun gHA04 